\title{
Cartan geometry of spacetimes with a nonconstant cosmological function $\Lambda$
}

\author{
Hendrik Jennen* \\ Instituto de Física Teórica, Universidade Estadual Paulista, Rua Dr. Bento Teobaldo Ferraz, 271, \\ 01140-070 São Paulo, São Paulo, Brazil
}

(Received 13 June 2014; revised manuscript received 18 September 2014; published 23 October 2014)

\begin{abstract}
We present the geometry of spacetimes that are tangentially approximated by de Sitter spaces whose cosmological constants vary over spacetime. Cartan geometry provides one with the tools to describe manifolds that reduce to a homogeneous Klein space at the infinitesimal level. We consider a Cartan geometry in which the underlying Klein space is at each point a de Sitter space, for which the combined set of pseudoradii forms a nonconstant function on spacetime. We show that the torsion of such a geometry receives a contribution that is not present for a cosmological constant. The structure group of the obtained de Sitter-Cartan geometry is by construction the Lorentz group $S O(1,3)$. Invoking the theory of nonlinear realizations, we extend the class of symmetries to the enclosing de Sitter group $S O(1,4)$, and compute the corresponding spin connection, vierbein, curvature, and torsion.
\end{abstract}

DOI: 10.1103/PhysRevD.90.084046

PACS numbers: 04.20.Cv, 02.40.-k, 98.80.-k

\section{INTRODUCTION}

In theories of gravity, the strong equivalence principle implies that spacetime $\mathcal{M}$ is locally approximated by the spacetime underlying special relativity [1]. Since the laws that govern special relativistic physics are covariant with respect to the Poincaré group $I S O(1,3)$, i.e., the semidirect product of the Lorentz group $S O(1,3)$ and the group of Poincaré displacements, the corresponding spacetime is the affine Minkowski space $M$. Although finite Poincaré translations are not defined for a generic spacetime, the equivalence principle indicates that locally they are in oneto-one correspondence with infinitesimal active diffeomorphisms, for both sets generate translations along spacetime [2]. Mathematically speaking, there is a 1-form, called the vierbein, which at any point is valued in the algebra of Poincaré translations $\mathfrak{t}=\mathfrak{i} \mathfrak{g} \mathfrak{v}(1,3) / \mathfrak{g} \mathfrak{v}(1,3)$. The vierbein pulls back or solders the geometric and algebraic structure of $t$ to spacetime. For example, the Minkowski metric on $t$ gives way to a metric of the same signature on $\mathcal{M}$, from which it follows that the vierbein can be chosen to be an orthonormal frame-an idealized observer-along spacetime. Due to the equivalence principle, Lorentz transformations of these observers constitute a symmetry and are therefore elements of the structure group of the geometry, which in turn leads to the introduction of a spin connection.

The right mathematical framework for the setting just outlined is due to Elie Cartan [3], in which the $\mathfrak{g} \mathfrak{v}(1,3)$ valued spin connection and the $\mathrm{t}$-valued vierbein are combined into an $\mathfrak{i} \mathfrak{g} \mathfrak{v}(1,3)$-valued Cartan connection, thereby defining a Riemann-Cartan geometry [2]. It is explained comprehensibly in [4] how the $\mathfrak{i} \mathfrak{g} \mathfrak{p}(1,3)$-valued connection gives a prescription for rolling without slipping the affine Minkowski space along the integral curves of

\footnotetext{
*hjennen@ift.unesp.br
}

vector fields on spacetime. It is indeed the central idea behind Cartan geometry that a homogeneous model space is generalized to a nonhomogeneous space, for which the local structure is algebraically isomorphic to the one of the model space [5], and where the degree of nonhomogeneity is quantified by the presence of curvature and torsion. In the manner thus explained, the choice for a Riemann-Cartan geometry to describe spacetimes underlying theories of gravity is implied by the equivalence principle, together with the assumption that the local kinematics are governed by the Poincaré group.

When the $\mathfrak{i} \mathfrak{g} \mathfrak{v}(1,3)$-valued Cartan connection is replaced by one that is valued in the de Sitter algebra $\mathfrak{g} \mathfrak{o}(1,4)$, spacetime is locally approximated by a de Sitter space $d S$ in place of the affine Minkowski space, a structure we shall call a de Sitter-Cartan geometry. Since the vierbein is valued in the space of de Sitter transvections $\mathfrak{p}=\mathfrak{g} \mathfrak{o}(1,4) / \mathfrak{g} \mathfrak{o}(1,3)$, translations in a de Sitter-Cartan spacetime are generated by elements of $\mathfrak{p}$. This implies that the commutator of infinitesimal translations is proportional to a Lorentz rotation. The constant of proportionality is essentially the cosmological constant of the tangent de Sitter spaces [4]. It is then sensible to identify this geometric cosmological constant with the dark energy on spacetime. Such an interpretation is in concordance with the MacDowell-Mansouri model for gravity [6]. In this model, the fundamental field is indeed a $\mathfrak{g} \mathfrak{o}(1,4)$-valued Cartan connection, for which the action is equivalent, up to topological terms, with the Palatini action for general relativity in the presence of a cosmological constant $[4,7]$.

At any point in a de Sitter-Cartan spacetime, the cosmological constant is related to a length scale defined in the commutation relations of the de Sitter transvections. Therefore, it is rather straightforward to generalize to geometries in which this length scale becomes a nonconstant function on spacetime. In Sec. II, we claim some 
originality for constructing a de Sitter-Cartan geometry that provides spacetime with a cosmological function $\Lambda$, which in general does not satisfy $d \Lambda=0$. We shall see that a nonconstant $\Lambda$ gives rise to a new term in the expression for the torsion of the de Sitter-Cartan geometry.

\section{DE SITTER-CARTAN GEOMETRY WITH A COSMOLOGICAL FUNCTION}

A de Sitter-Cartan geometry is the Cartan geometry modeled on $(\mathfrak{g} \mathfrak{p}(1,4), S O(1,3))$, which means it consists of a principal Lorentz bundle $P(\mathcal{M}, S O(1,3))$ over spacetime, on which is defined a $\mathfrak{g} \mathfrak{o}(1,4)$-valued Cartan connection $A$. For a rigorous discussion on Cartan geometry, see $[5,8]$, while the articles $[4,7,9]$ are very helpful to develop an intuition that goes along with the mathematics. The connection $A$ provides spacetime with the information that it is tangentially approximated by de Sitter space, the homogeneous Klein space with respect to which inhomogeneities are measured [10]. We shall construct a de SitterCartan geometry, in which these tangent de Sitter spaces have cosmological constants that are not required to be the same over spacetime. As a consequence, the thus obtained geometry describes a manifold with arbitrary curvature and torsion on which a nonconstant cosmological function $\Lambda$ is defined from the onset.

Under the action of a local $S O(1,3)$ transformation $h$, the de Sitter-Cartan connection transforms according to [5]

$$
A \mapsto \operatorname{Ad}(h)(A+d) .
$$

The connection is valued in the de Sitter algebra $\mathfrak{s} \mathfrak{o}(1,4)$, which is characterized by the commutation relations

$$
\begin{aligned}
-i\left[M_{a b}, M_{c d}\right] & =\eta_{a c} M_{b d}-\eta_{a d} M_{b c}+\eta_{b d} M_{a c}-\eta_{b c} M_{a d}, \\
-i\left[M_{a b}, P_{c}\right] & =\eta_{a c} P_{b}-\eta_{b c} P_{a}, \\
-i\left[P_{a}, P_{b}\right] & =-l^{-2} M_{a b},
\end{aligned}
$$

where $\eta_{a b}=(+,-,-,-)$, while we parametrize an element of $\mathfrak{g} \mathfrak{o}(1,4)$ by $\frac{i}{2} \lambda^{a b} M_{a b}+i \lambda^{a} P_{a}$. The reductive nature of the algebra schematically reads as

$$
\mathfrak{s} \mathfrak{o}(1,4)=\mathfrak{g} \mathfrak{v}(1,3) \oplus \mathfrak{p}
$$

where $\mathfrak{S} \mathfrak{o}(1,3)=\operatorname{span}\left\{M_{a b}\right\}$ is the Lorentz subalgebra and $\mathfrak{p}=\operatorname{span}\left\{P_{a}\right\}$ the subspace of infinitesimal de Sitter transvections or translations. The latter are defined by $P_{a}=M_{a 4} / l$, where $l$ is an a priori arbitrary length scale that effectively determines the cosmological constant of the corresponding Klein geometry $d S=S O(1,4) / S O(1,3)$, namely, [4]

$$
\Lambda=\frac{3}{l^{2}}
$$

Since the Cartan connection is at any point $x \in \mathcal{M}$ valued in a copy of $\mathfrak{g} \mathfrak{o}(1,4)$, we may choose the set of length scales $l(x)$ to form a smooth function. Doing so, the cosmological constants of the corresponding tangent de Sitter spaces also constitute a generic nonconstant cosmological function $\Lambda(x)$ on spacetime. In the following paragraphs we discuss the implications of a nonconstant $\Lambda$ for the de Sitter-Cartan geometry.

Corresponding to the reductive splitting (3), we decompose the Cartan connection and its curvature $F=d A+$ $\frac{1}{2}[A, A]$ as

$$
A=\frac{i}{2} A^{a b} M_{a b}+i A^{a} P_{a} \quad \text { and } \quad F=\frac{i}{2} F^{a b} M_{a b}+i F^{a} P_{a},
$$

from which it follows that $A^{a}$ and $F^{a}$ have the dimension of length. The $\mathfrak{g} \mathfrak{o}(1,3)$-valued 1-form $A^{a b}$ is an Ehresmann connection for local Lorentz transformations [8], i.e., a spin connection, while the forms $A^{a}$ constitute a vierbein. Note that the decompositions (5) are well defined, since local Lorentz transformations leave the reductive splitting invariant. Due to the presence of a spin connection and vierbein, it is possible to define local Lorentz and diffeomorphism covariant differentiation, as well as a metric structure on spacetime; see, e.g., [11].

Given the commutation relations (2), one computes the curvature $F^{a b}$ and torsion $F^{a}$ in terms of the spin connection and vierbein:

$$
\begin{aligned}
F^{a b} & =d A^{a b}+A^{a}{ }_{c} \wedge A^{c b}+\frac{1}{l^{2}} A^{a} \wedge A^{b} \\
& =d_{A} A^{a b}+\frac{1}{l^{2}} A^{a} \wedge A^{b}, \\
F^{a} & =d A^{a}+A^{a}{ }_{b} \wedge A^{b}-\frac{1}{l} d l \wedge A^{a} \\
& =d_{A} A^{a}-\frac{1}{l} d l \wedge A^{a} .
\end{aligned}
$$

In the limit of an everywhere diverging length scale $l$, or equivalently, an everywhere vanishing cosmological constant, the expressions (6) reduce to the curvature $d_{A} A^{a b}$ and torsion $d_{A} A^{a}$ for a Riemann-Cartan geometry [11]. In the generic case, however, the curvature and torsion are not given by the exterior covariant derivatives of the spin connection and vierbein. The extra term in (6a) represents the curvature of the local de Sitter space. This contribution is present because the commutator of two infinitesimal de Sitter transvections equals an element of the Lorentz algebra. In addition, there is a new term in the expression (6b) for the torsion if the length scale is a nonconstant function. This term comes about as follows. The torsion is the $\mathfrak{p}$-valued 2-form $F_{\mathfrak{p}}=d A_{\mathfrak{p}}+\left[A_{\mathfrak{h}}, A_{\mathfrak{p}}\right]$ [5], with $\mathfrak{h}=$ $\mathfrak{S o}(1,3)$. The first term in this expression is expanded as 


$$
d A_{\mathfrak{p}}=d\left(i A^{a} P_{a}\right)=i d A^{a} P_{a}-i\left(\frac{d l}{l} \wedge A^{a}\right) P_{a},
$$

since $P_{a}=M_{a 4} / l$. By use of the relation (4) between $l$ and the cosmological function $\Lambda$, the last term of the torsion can be rewritten as

$$
-d \ln l \wedge A^{a}=\frac{1}{2} d \ln \Lambda \wedge A^{a},
$$

which shows that this contribution depends on the relative infinitesimal change of the cosmological function along spacetime, rather than on its absolute change.

Although the curvature and torsion have contributions that are not there for a Riemann-Cartan geometry, the Bianchi identities are unchanged:

$$
\begin{aligned}
d_{A} \circ d_{A} A^{a b} & \equiv 0, \\
d_{A} \circ d_{A} A^{a}+A^{b} \wedge d_{A} A_{b}{ }^{a} & \equiv 0,
\end{aligned}
$$

where $d_{A}$ is the exterior covariant derivative with respect to the spin connection.

The transformations that are consistent with the given geometry are local Lorentz transformations and spacetime diffeomorphisms, the latter being unphysical as they merely relabel spacetime coordinates [12]. In contrast, with respect to elements of $S O(1,4)$, we see from (5) that the spin connection and vierbein, and the torsion and curvature form irreducible multiplets. Due to the reductive nature of $\mathfrak{s} \mathfrak{o}(1,4)$, these geometric objects are well defined up to local Lorentz transformations only. Since local translational symmetry may play an important role in theories of gravity, there is the need to extend the structure group to $S O(1,4)$, while preserving the presence of these different objects, necessary to construct geometric theories of gravity. This will be discussed for the given de Sitter-Cartan geometry in the following section.

\section{III. $S O(1,4)$-INVARIANT DE SITTER-CARTAN GEOMETRY WITH A COSMOLOGICAL FUNCTION}

In order to extend the structure group to $S O(1,4)$ and have geometric objects that are well defined through the decomposition of a Cartan connection and curvature according to the reductive splitting (3), we nonlinearly realize the de Sitter-Cartan connection of Sec II. To realize connections on spacetime in a nonlinear way was first considered by Stelle and West $[13,14]$, while its usefulness for theories of gravity has been pointed out in, e.g., [15-18]. The formalism of nonlinear realizations was developed to systematically study spontaneous symmetry breaking in phenomenological field theory [19-21], in which linearly transforming irreducible multiplets become nonlinear but reducible realizations, when the symmetry group is realized nonlinearly by one of its subgroups.

A Cartan connection on a principal Lorentz bundle $P$ may be thought of as an Ehresmann connection on a principal $S O(1,4)$ bundle $Q$ over $\mathcal{M}$ that is reduced to $P$ [5]. This is in essence a symmetry breaking process [15], for the reason that it corresponds to singling out a section $\xi$ of the associated bundle $Q \times_{S O(1,4)} d S$ of tangent de Sitter spaces, thereby reducing the structure group $S O(1,4)$ pointwise to $S O(1,3)_{\xi}$, the isotropy group of the point $\xi(x)$ in the internal de Sitter space $d S_{x}$ [22,23]. Most importantly, the reduction is not canonical, i.e., the section $\xi$ can be chosen arbitrarily, and the broken symmetries are nonmanifestly restored by realizing them nonlinearly through elements of the Lorentz group. Consequently, decomposing a nonlinear de Sitter-Cartan connection according to the reductive splitting of $\mathfrak{s} \mathfrak{o}(1,4)$ gives way to true geometric objects, well defined with respect to all elements of $S O(1,4)$.

Before we construct a nonlinear de Sitter-Cartan geometry with a nonconstant cosmological function, we recall a handful of facts on nonlinear realizations for the de Sitter group; see also $[24,25]$. Within some neighborhood of the identity, an element $g$ of $S O(1,4)$ can uniquely be represented in the form

$$
g=\exp (i \xi \cdot P) \tilde{h}
$$

with $\tilde{h} \in S O(1,3)$ and $\xi \cdot P=\xi^{a} P_{a}$. The $\xi^{a}$ parametrize the coset space $S O(1,4) / S O(1,3)$ so that they constitute a coordinate system for de Sitter space. This parametrization allows us to define the action of $S O(1,4) \ni g_{0}$ on de Sitter space by

$$
g_{0} \exp (i \xi \cdot P)=\exp \left(i \xi^{\prime} \cdot P\right) h^{\prime} ; \quad h^{\prime}=\tilde{h}^{\prime} \tilde{h}^{-1},
$$

where $\xi^{\prime}=\xi^{\prime}\left(g_{0}, \xi\right)$ and $h^{\prime}=h^{\prime}\left(g_{0}, \xi\right)$ are in general nonlinear functions of the indicated variables. In case $g_{0}=h_{0}$ is an element of $S O(1,3)$, the action is linear and the transformation of $\xi$ is given explicitly by

$$
h_{0}: i \xi \cdot P \mapsto i \xi^{\prime} \cdot P=i \xi \cdot \operatorname{Ad}\left(h_{0}\right)(P) .
$$

If on the other hand $g_{0}=1+i \epsilon \cdot P$ is an infinitesimal pure de Sitter translation, the variations $\delta \xi^{a}$ and $\delta h^{a b}$ satisfy

$$
\begin{aligned}
& \exp (-i \xi \cdot P) i \epsilon \cdot P \exp (i \xi \cdot P)-\exp (-i \xi \cdot P) \delta \exp (i \xi \cdot P) \\
& \quad=\frac{i}{2} \delta h \cdot M
\end{aligned}
$$

where $\frac{i}{2} \delta h \cdot M=h^{\prime}-1 \in \mathfrak{s} \mathfrak{o}(1,3)$ and $\delta h \cdot M=\delta h^{a b} M_{a b}$. This equation is solved by 


$$
\begin{aligned}
\delta \xi^{a} & =\epsilon^{a}+\left(\frac{z \cosh z}{\sinh z}-1\right)\left(\epsilon^{a}-\frac{\xi^{a} \epsilon_{b} \xi^{b}}{\xi^{2}}\right), \\
\delta h^{a b} & =\frac{1}{l^{2}} \frac{\cosh z-1}{z \sinh z}\left(\epsilon^{a} \xi^{b}-\epsilon^{b} \xi^{a}\right),
\end{aligned}
$$

where we made use of the notation $z=l^{-1} \xi$ and $\xi=\left(\eta_{a b} \xi^{a} \xi^{b}\right)^{1 / 2}$.

Subsequently, let $\psi$ be a field that belongs to some linear representation $\sigma$ of $S O(1,4)$. Given a local section of the associated bundle of homogeneous de Sitter spaces, i.e., $\xi: U \subset \mathcal{M} \rightarrow U \times d S$, the corresponding nonlinear field is constructed pointwise as

$$
\bar{\psi}(x)=\sigma(\exp (-i \xi(x) \cdot P)) \psi(x)
$$

Under a local de Sitter transformation $g_{0}$, it rotates only according to its $S O(1,3)$ indices, namely, $\bar{\psi}^{\prime}(x)=$ $\sigma\left(h^{\prime}\left(\xi, g_{0}\right)\right) \bar{\psi}(x)$. It is manifest that the irreducible linear representation $\psi$ has given way to a nonlinear and reducible realization $\bar{\psi}$.

In concordance with the prescription (10) to construct nonlinear realizations, the nonlinear $\mathfrak{g} \mathfrak{o}(1,4)$-valued Cartan connection is defined as [14]

$$
\bar{A}=\operatorname{Ad}(\exp (-i \xi \cdot P))(A+d)
$$

Under local de Sitter transformations, the field $\bar{A}$ transforms according to

$$
\bar{A} \mapsto \operatorname{Ad}\left(h^{\prime}\left(\xi, g_{0}\right)\right)(\bar{A}+d)
$$

Because elements of $S O(1,4)$ are nonlinearly realized as elements of $S O(1,3)$, the reductive decomposition $\bar{A}_{\mathfrak{h}}+\bar{A}_{\mathfrak{p}}$ is invariant under local de Sitter transformations. It is then sensible to define the spin connection and vierbein through these projections as $\omega=\bar{A}_{\mathfrak{h}}$ and $e=\bar{A}_{\mathfrak{p}}$, respectively.

The spin connection $\omega$ and vierbein $e$ can be expressed in terms of the section $\xi$ and the projections $A_{\mathfrak{h}}$ and $A_{\mathfrak{p}}$ of the linear $S O(1,4)$ connection. These relations follow from (11), in which the different objects appear according to

$$
\begin{aligned}
& \frac{i}{2} \omega^{a b} M_{a b}+i e^{a} P_{a} \\
& \quad=\operatorname{Ad}(\exp (-i \xi \cdot P))\left(\frac{i}{2} A^{a b} M_{a b}+i A^{a} P_{a}+d\right) .
\end{aligned}
$$

To carry out the computation of the right-hand side we utilize the techniques of [14,24], explained in their appendices. In short, one expands the adjoint action of the exponential as a power series in the adjoint action of its generating element $-i \xi \cdot P$. The latter is just the Lie commutator and is given explicitly in (2). We find

$$
\begin{aligned}
\omega^{a b}= & A^{a b}-\frac{\cosh z-1}{l^{2} z^{2}}\left[\xi^{a}\left(d \xi^{b}+A^{b}{ }_{c} \xi^{c}\right)\right. \\
& \left.-\xi^{b}\left(d \xi^{a}+A^{a}{ }_{c} \xi^{c}\right)\right]-\frac{\sinh z}{l^{2} z}\left(\xi^{a} A^{b}-\xi^{b} A^{a}\right),
\end{aligned}
$$

$$
\begin{aligned}
e^{a}= & A^{a}+\frac{\sinh z}{z}\left(d \xi^{a}+A^{a}{ }_{b} \xi^{b}\right)-\frac{d l}{l} \xi^{a} \\
& +(\cosh z-1)\left(A^{a}-\frac{\xi^{b} A_{b} \xi^{a}}{\xi^{2}}\right) \\
& -\left(\frac{\sinh z}{z}-1\right) \frac{\xi^{b} d \xi_{b} \xi^{a}}{\xi^{2}} .
\end{aligned}
$$

These expressions are almost identical to the corresponding objects found by Stelle and West [14]. The difference to note is that we have a new term in the expression (12b) for the vierbein, namely, $-l^{-1} d l \xi^{a}$. This term is present because it is possible that the internal de Sitter spaces are characterized by cosmological constants that are not necessarily equal along spacetime. More precisely, one has to take into account the possibility that the in $\mathfrak{p}$ defined length scale is a nonconstant function; see Sec. II. On the other hand, the results of [14] specialize for the case that the local de Sitter spaces have the same pseudoradius at any point in spacetime. When $l$ is a constant function, one naturally recovers the results of [14].

Upon the action of local de Sitter transformations, the linear curvature $F$ rotates in the adjoint representation. Therefore, one deduces that the nonlinear Cartan curvature $\bar{F}$ is equal to the exterior covariant derivative of the nonlinear connection, i.e.,

$$
\bar{F}=\operatorname{Ad}(\exp (-i \xi \cdot P))(F)=d \bar{A}+\frac{1}{2}[\bar{A}, \bar{A}]
$$

which complies with the structure of a Cartan geometry. The nonlinear Cartan curvature is a $\mathfrak{g} \mathfrak{v}(1,4)$-valued 2-form on spacetime, which we decompose once again according to $\bar{F}=\bar{F}_{\mathfrak{h}}+\bar{F}_{\mathfrak{p}}$. Since $\bar{F}$ transforms-in general, nonlinearly_with elements of $S O(1,3)$, the reductive splitting is invariant under local de Sitter transformations. This suggests that $\bar{F}_{\mathfrak{h}}$ and $\bar{F}_{\mathfrak{p}}$ must be considered the genuine curvature and torsion of the Cartan geometry, which are denoted by $R$ and $T$, respectively. The definition (13) implies that

$$
\begin{aligned}
& \frac{i}{2} R^{a b} M_{a b}+i T^{a} P_{a} \\
& \quad=\operatorname{Ad}(\exp (-i \xi \cdot P))\left(\frac{i}{2} F^{a b} M_{a b}+i F^{a} P_{a}\right)
\end{aligned}
$$

from which one is able to express the curvature and torsion in terms of $\xi, F_{\mathfrak{h}}$ and $F_{\mathfrak{p}}$ : 


$$
\begin{aligned}
R^{a b}= & F^{a b}-\frac{\cosh z-1}{l^{2} z^{2}} \xi^{c}\left(\xi^{a} F^{b}{ }_{c}-\xi^{b} F^{a}{ }_{c}\right) \\
& -\frac{\sinh z}{l^{2} z}\left(\xi^{a} F^{b}-\xi^{b} F^{a}\right), \\
T^{a}= & \frac{\sinh z}{z} \xi^{b} F_{b}^{a}+\cosh z F^{a}+(1-\cosh z) \frac{\xi_{b} F^{b} \xi^{a}}{\xi^{2}} .
\end{aligned}
$$

From (13) it furthermore follows that

$R^{a b}=d_{\omega} \omega^{a b}+\frac{1}{l^{2}} e^{a} \wedge e^{b} \quad$ and $\quad T^{a}=d_{\omega} e^{a}-\frac{1}{l} d l \wedge e^{a}$

These equations, which express the curvature and torsion in terms of the spin connection and vierbein, are the ones expected for a Cartan geometry. Because the exterior covariant derivative of $\bar{F}$ is always zero, there are two Bianchi identities that are formally the same as those given by (7), i.e.,

$$
d_{\omega} \circ d_{\omega} \omega^{a b} \equiv 0 \quad \text { and } \quad d_{\omega} \circ d_{\omega} e^{a}+e^{b} \wedge d_{\omega} \omega_{b}{ }^{a} \equiv 0 .
$$

When the section $\xi$ is gauge fixed along spacetime, and for convenience at any point is chosen the origin of the tangent de Sitter spaces, i.e., $\xi^{a}(x)=0$, all the expressions reduce to those of Sec. II. This is to be expected, because the broken symmetries are not considered, and the geometry is described simply by a $S O(1,4)$ Ehresmann connection for which only $S O(1,3)$ transformations-the isotropy group of $\xi^{a}=0$-are taken into account. This has precisely been the way in which the de Sitter-Cartan geometry of Sec. II was set up.

Finally, let us remark that if $A^{a b}$ and $A^{a}$ can be made to vanish everywhere, so that also $F^{a b}$ and $F^{a}$ are equal to zero, it follows that

$$
R^{a b}=0 \quad \text { and } \quad T^{a}=0 .
$$

This shows that the nonhomogeneity of $\mathcal{M}$ is encoded in $A$ and $F$, and naturally independent of the section $\xi$.

\section{CONCLUSIONS AND OUTLOOK}

In this work we have generalized the geometric framework of de Sitter-Cartan spacetimes with a cosmological constant to the case of a nonconstant cosmological function $\Lambda$. A de Sitter-Cartan spacetime consists of a principal Lorentz bundle over spacetime, on which is defined a $\mathfrak{S} \mathfrak{o}(1,4)$-valued Cartan connection. It accounts for a spin connection and vierbein, as well as for their curvature and torsion, whereas spacetime is locally approximated by de Sitter spaces. The cosmological constants of these tangent de Sitter spaces are determined by a length scale, defined in the translational part of $\mathfrak{s} \mathfrak{v}(1,4)$. By letting this length scale depend arbitrarily on the spacetime point in Sec. II, we obtained a de SitterCartan geometry that accommodates a cosmological function by construction. Most importantly, it was shown that a nonconstant $\Lambda$ gives rise to an extra contribution in the expression for the torsion, in which the cosmological function appears through its logarithmic derivative. In the limit $\Lambda \rightarrow 0$ one recovers the well-known RiemannCartan spacetime with arbitrary curvature and torsion. In Sec. III, the de Sitter-Cartan connection has been realized nonlinearly in order to obtain $S O(1,4)$-covariant definitions for the spin connection and vierbein, and likewise for the curvature and torsion. This generalized previous results to include a nonconstant $\Lambda$.

The cosmological function could be used to model dark energy that changes along space and time, in which way it might give an alternative description for one of the models for time-evolving dark energy [26,27]. To determine the value of the cosmological function along spacetime, an adequate action for the gravitational field coupled to matter will have to be defined. By including invariants of the torsion tensor on the gravitational side, the first derivative of the cosmological function will automatically be present. Matter fields can be coupled both minimally to the gravitational field and in another nonminimal way. The analysis of such models that make use of the framework outlined in this paper is an interesting and important subject of future research. Since the cosmological function quantifies the lack of commutation of two infinitesimal spacetime translations, the local kinematics on spacetime depend on $\Lambda$. Consequently, there would be a link between the dynamical character of the cosmological function and its kinematical implications.

Another point of interest comes about upon noting that, when the de Sitter algebra is contracted to the Poincaré algebra, namely, when $l \rightarrow \infty$ in the commutation relations (2), the geometric objects of Sec. III reduce to those of teleparallel gravity $[28,29]$. This observation suggests that the geometry of spacetime that underlies teleparallel gravity is described by a Riemann-Cartan geometry (with vanishing curvature), for which the Poincare translations are realized nonlinearly as elements of $S O(1,3)$. In fact, from (9) one sees that the nonlinear element of the Lorentz algebra, which corresponds to an infinitesimal Poincaré translation with parameters $\epsilon^{a}$, vanishes, for

$$
\delta h^{a b}=\lim _{l \rightarrow \infty} \frac{1}{l^{2}} \frac{\cosh z-1}{z \sinh z}\left(\epsilon^{a} \xi^{b}-\epsilon^{b} \xi^{a}\right)=0 .
$$

One then concludes that any Poincare translation is trivially realized by the identity transformation, a property that is relied upon in the interpretation of teleparallel gravity as a gauge theory for the Poincaré translations. Given the knowledge that the geometric structure of teleparallel 
gravity is such a Riemann-Cartan spacetime, the de SitterCartan geometry of Sec. III might be the right framework to generalize teleparallel gravity to a theory that is invariant under local $S O(1,4)$ transformations, in place of the elements of the Poincaré group.

\section{ACKNOWLEDGMENTS}

The author would like to thank J.G. Pereira for the helpful discussions and suggestions. He also gratefully acknowledges financial support by Coordenação de Aperfeiçoamento de Pessoal de Nível Superior (CAPES).
[1] E. Di Casola, S. Liberati, and S. Sonego, arXiv:1310.7426.

[2] F. W. Hehl, P. Von Der Heyde, G. D. Kerlick, and J. M. Nester, Rev. Mod. Phys. 48, 393 (1976).

[3] E. Cartan, Acta Math. 48, 1 (1926).

[4] D. K. Wise, Classical Quantum Gravity 27, 155010 (2010).

[5] R. W. Sharpe, Differential Geometry: Cartan's Generalization of Klein's Erlangen Program (Springer, New York, 1997).

[6] S. W. MacDowell and F. Mansouri, Phys. Rev. Lett. 38, 739 (1977).

[7] H. F. Westman and T. G. Zlosnik, arXiv:1203.5709.

[8] D. V. Alekseevsky and P. W. Michor, Publ. Math. Debrecen 47, 349 (1995).

[9] D. K. Wise, Symmetry Integrability Geom. Methods Appl. 5, 080 (2009).

[10] F. Klein, Bull. N.Y. Math. Soc. 2, 215 (1893).

[11] T. Ortín, Gravity and Strings (Cambridge University Press, Cambridge, England, 2004).

[12] J. D. Edelstein and J. Zanelli, J. Phys. Conf. Ser. 33, 83 (2006).

[13] K. S. Stelle and P. C. West, J. Phys. A 12, L205 (1979).

[14] K. S. Stelle and P. C. West, Phys. Rev. D 21, 1466 (1980).

[15] D. K. Wise, J. Phys. Conf. Ser. 360, 012017 (2012).

[16] A. Tiemblo and R. Tresguerres, Recent Res. Dev. Phys. Chem. 5, 1255 (2004).
[17] R. Tresguerres, Int. J. Geom. Methods Mod. Phys. 05, 171 (2008).

[18] F. W. Hehl and M. Blagojevic, Gauge Theories of Gravitation (Imperial College Press, London, 2013).

[19] S. Coleman, J. Wess, and B. Zumino, Phys. Rev. 177, 2239 (1969).

[20] C. G. Callan, Jr., S. Coleman, J. Wess, and B. Zumino, Phys. Rev. 177, 2247 (1969).

[21] D. V. Volkov, Sov. J. Part. Nucl. 4, 3 (1973).

[22] D. Husemöller, Fibre Bundles (Springer, New York, 1966).

[23] G. W. Gibbons and S. Gielen, Classical Quantum Gravity 26, 135005 (2009).

[24] B. Zumino, Nucl. Phys. B127, 189 (1977).

[25] J. Wess and J. Bagger, Supersymmetry and Supergravity (Princeton University, Princeton, NJ, 1992).

[26] P. J. E. Peebles and B. Ratra, Rev. Mod. Phys. 75, 559 (2003).

[27] E. J. Copeland, M. Sami, and S. Tsujikawa, Int. J. Mod. Phys. D 15, 1753 (2006).

[28] R. Aldrovandi and J. G. Pereira, Teleparallel Gravity: An Introduction, Fundamental Theories of Physics Vol. 173 (Springer, New York, 2012).

[29] H. I. Arcos and J. G. Pereira, Int. J. Mod. Phys. D 13, 2193 (2004). 